\title{
GPR-Based Landmine Detection and Identification Using Multiple Features
}

\author{
Kwang Hee Ko, Gyubin Jang, Kyungmi Park, and Kangwook Kim \\ The School of Mechatronics, Gwangju Institute of Science and Technology, 123 Cheomdan-gwagiro, Buk-gu, \\ Gwangju 500-712, Republic of Korea \\ Correspondence should be addressed to Kwang Hee Ko, khko@gist.ac.kr
}

Received 1 May 2012; Revised 17 September 2012; Accepted 18 September 2012

Academic Editor: Tat Yeo

Copyright ( 92012 Kwang Hee Ko et al. This is an open access article distributed under the Creative Commons Attribution License, which permits unrestricted use, distribution, and reproduction in any medium, provided the original work is properly cited.

This paper presents a method to identify landmines in various burial conditions. A ground penetration radar is used to generate data set, which is then processed to reduce the ground effect and noise to obtain landmine signals. Principal components and Fourier coefficients of the landmine signals are computed, which are used as features of each landmine for detection and identification. A database is constructed based on the features of various types of landmines and the ground conditions, including the different levels of moisture and types of ground and the burial depths of the landmines. Detection and identification is performed by searching for features in the database. For a robust decision, the counting method and the Mahalanobis distancebased likelihood ratio test method are employed. Four landmines, different in size and material, are considered as examples that demonstrate the efficiency of the proposed method for detecting and identifying landmines.

\section{Introduction}

Landmine removal is a critical problem faced by many countries around the world, and the situation can be compounded by natural disasters or land development. Therefore, it is an urgent issue to detect landmines in the ground and remove them safely. The process of landmine removal starts with the detection of landmines in the ground. For safe detection, non-touch-based detection methods are required. These methods involve the detection of landmines in the signals obtained by non-touch-based sensors, such as metal detectors and radars. Among those sensors, ground penetrating radars, or GPRs, are an attractive choice for landmine detection due to their advantages over other sensors. The GPR can be used as a stand-alone sensor or as a complementary sensor to a metal detector $[1,2]$. It can detect both metal and nonmetal landmines [3]. Moreover, its weight can be made light, so that it can be installed in a handheld system or in a vehicle-mounted system in the form of an array of multiple antenna elements [4-6].
In the landmine detection step, the key factor is to obtain unique signatures of a landmine from the signal, which are used as tags of each landmine. The signal may be, however, contaminated by noise, surface reflections, and so forth. Therefore, minimizing such influences and extracting the unique signatures for each landmine is an active research topic. Many studies show various methods of computing signatures from GPR data [7-12]. Tantum et al. [13] compare different algorithms for landmine detection. Each approach, however, utilizes one feature for detection, which may not be enough for robust detection because the discernibility of a single feature could be compromised by disturbances to the signal. This problem can be avoided with multiple features extracted by different methods from a signal using by sensor with no parameter changes. These features reflect different aspects of a landmine signal and would enhance their discernibility to a large extent. One paper [14] presents feature fusion, which includes either features obtained by different sensors or features obtained by one sensor with different parameter settings. This concept, 
however, is not useful because it requires different sensors or one sensor with different parameter settings, which is not a practical configuration.

The landmine detection step may be followed by landmine identification, which is a process to identify the types of landmines and their burial depths. This additional information can help detect and remove landmines more efficiently. Identification involves more steps than detection and has not been significantly investigated in the related field.

In this study, the problem of detection and identification of landmines using GPRs is addressed, and a novel method of detection and identification using multiple features is proposed. Principal component analysis and Fourier Transform are employed to obtain eigenvalues and Fourier coefficients, respectively, which are used as features associated with each landmine. Algorithms for detection and identification using multiple features are presented and tested with various examples.

\section{Overall Process}

The overall process of the proposed pipeline of landmine detection and identification is illustrated in Figure 1. The input of the process is the GPR signal, and part of the signal containing landmine effects is extracted after the noise and ground effects are reduced. The principal component analysis (PCA) and Fourier Transform (FT) methods compute features from the landmine signal. A database is constructed by storing the features with the associated landmine information. This database construction uses experimental data with known landmine information. For detection and identification, the input signal is processed to generate features, and similar features are identified in the database. The decision for detection and identification employs two methods: the counting value method first performs detection, followed by identification by the likelihood ratio test method. Once a landmine is successfully identified, the buried depth of the landmine is estimated. In the subsequent sections, the theories and algorithms of each step are introduced.

2.1. Hardware and GPR Signals. The GPR system used in this work has a GPR antenna at the end of an arm that moves horizontally, covering the scanning area, as shown in Figure 2. The specification of the GPR is summarized in Table 1 .

Targets are buried at different depths in a $2 \mathrm{~m} \times 1 \mathrm{~m} \times$ $1 \mathrm{~m}$ wooden box. The types of ground, the moisture levels of the ground, and the antenna heights and the target depths are changed for each scan. The antenna acquires 256 depth data samples at each location and scans the survey area to yield a dense data set, as shown in Figure 3. Next, all the data are regulated into 150 by 253 matrices with $5 \mathrm{~mm}$ intervals. The rows and the columns of the matrices indicate sampling locations and depths, respectively.

2.2. Reduction of Noise and Ground Effects. The GPR data (D) are assumed to be the sum of separate signals consisting of three components [7]: ground (G), landmine (L), and noise



FIGURE 1: The overall process of landmine detection and identification.

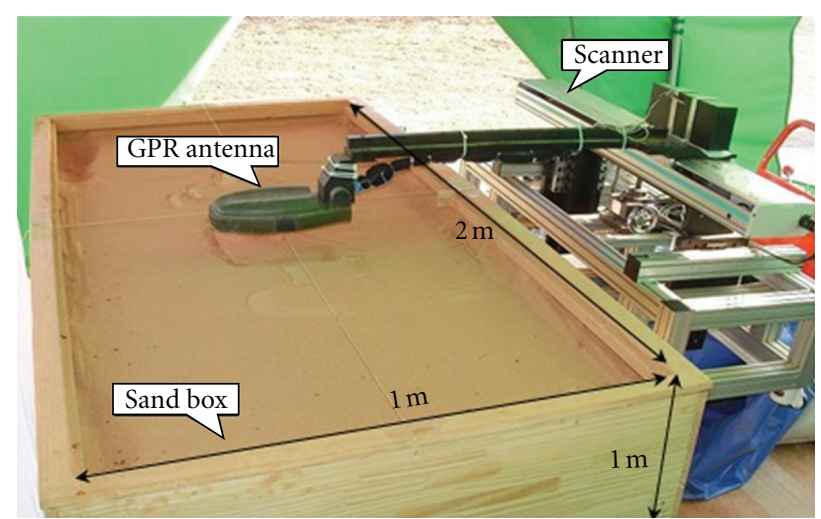

FIgURE 2: The GPR system used in the experiment.

(N). Therefore, it is represented by $\mathbf{D}=\mathbf{G}+\mathbf{L}+\mathbf{N}$. From this model, the signal $\mathbf{L}$ is obtained by subtracting $\mathbf{G}+\mathbf{N}$ from $\mathbf{D}$. Scanning a region without a landmine would generate $\mathbf{G}+\mathbf{N}$. Although this method is conceptually straightforward, there are two problems: one is that the signal should be normalized to eliminate the radar power difference for each scan, and 


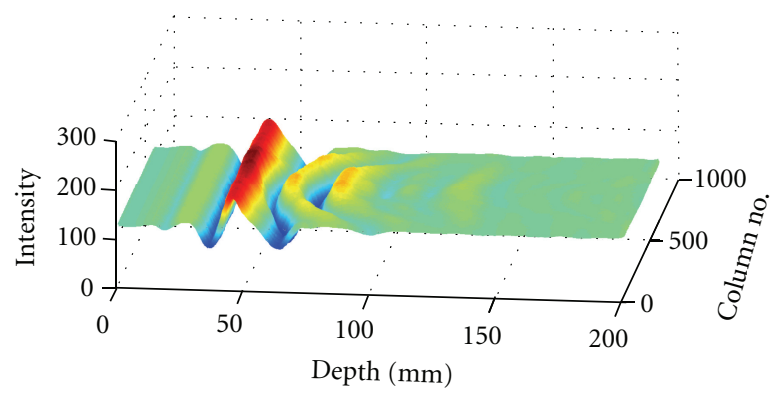

(a)



(b)

FIgURe 3: An example of raw GPR dat.

TABLE 1: The specification of the GPR used for the test.

\begin{tabular}{lc}
\hline Property & Value \\
\hline Internal pulse duration & $240 \mathrm{ps}(-6 \mathrm{~dB}$ width $)$ unipolar \\
Internal pulse amplitude & $15 \mathrm{~V}$ Peak \\
Pulse repetition frequencies & $1 \mathrm{MHz}$ \\
Bandwidth of operation & $250 \mathrm{MHz}$ to $2.5 \mathrm{GHz}$ \\
EIRP & $<-41 \mathrm{dBm} / \mathrm{MHz}$ \\
Radar sampling & 512 samples per scan \\
Receiver time range & $19.2 \mathrm{~ns}$ \\
Receiver sampling interval & $37.5 \mathrm{ps}$ \\
Output scan rate & $61 \mathrm{~Hz}$ \\
Sweep speed & $<1.5 \mathrm{~ms}{ }^{-1}$ \\
\hline
\end{tabular}

the other is that maintaining the same ground condition with and without a landmine is nearly impossible. Therefore, the approach cannot yield L consistently. An alternative approach is to select the reference signal, a signal obtained from $\mathbf{D}$ with the least amount of landmine influences, and to subtract it from $\mathbf{D}$. The reference signal is computed as the average of two columns of data far away from the column of the largest strength. This approach is advantageous in that there is no need to consider the ground condition and the radar signal normalization to obtain the $\mathbf{G}+\mathbf{N}$ signal since it is obtained in the same data set, eliminating the need for normalization. One example of GPR data is shown in Figure 3. The reference signal is subtracted to yield $\mathbf{L}$, which is shown in Figure 4.

2.3. Normalization. Once the landmine signal (L) is obtained, the intensity of the signal is normalized to reduce the influence caused by the difference of signal strength. The maximum and minimum intensity values, $I_{\max }$ and $I_{\min }$, are selected from the signal $\mathbf{L}$, which are then mapped to the values of $N_{\max }$ and $N_{\min }$, the limiting values for normalization. Then the normalized signal $I_{\text {normalized }}$ is computed by

$$
I_{\text {normalized }}=\left(\frac{I-I_{\min }}{I_{\max }-I_{\min }}\right) \times\left(N_{\max }-N_{\min }\right)+N_{\min } .
$$

Here, $I$ is an intensity value of $\mathbf{L}$.

\section{Feature Extraction and Database Construction}

Landmines are made of various materials, such as plastics and metals, and are different in size and shape. Therefore, they respond differently and bear unique signatures. In this work, two methods are used to extract such features from the signal L: principal component analysis (PCA) and Fourier Transform (FT).

3.1. Principal Component Analysis. PCA is a statistical method that can provide patterns of scattered data, $N$, in $K$ dimension. It constructs a $K \times K$ covariance matrix from the data. The eigenvectors and eigenvalues of the matrix are $\mathbf{E}_{c}$ $=\left(\mathbf{e}_{1}, \mathbf{e}_{2}, \ldots, \mathbf{e}_{K}\right)$ and $\mathbf{E}_{v}=\left(e_{1}, e_{2}, \ldots, e_{K}\right)$, respectively. $\mathrm{A}$ value of $e_{i}$ indicates how strong a pattern the data shows in the $\mathbf{e}_{i}$ direction. Using this property, a signal of a landmine would contain eigenvalues and eigenvectors that are unique to the landmine. In this work, each GPR data point in 3D space as shown in Figure 4 is projected onto the $X Z$ plane to generate a set of points in the $2 \mathrm{D}$ space as shown in Figure 5. Here, the $X$ axis indicates the column number and $Z$ is the intensity. Then the data in the $X Z$ plane are processed to compute two principal components $(N=2)$. Therefore two eigenvalues and eigenvectors are obtained, which can be considered as features of each landmine. Nondimensional eigenvectors could be considered for GPR landmine discrimination in the similar way as [15]. As the GPR data show, however, the eigenvectors would not be used for GPR landmine discrimination in this work. Most of the GPR data are clustered near the zero intensity and one principal component should follow that distribution pattern as $\mathbf{e}_{1}$ in Figure 5. Therefore, the eigenvectors for different GPR data show little difference and cannot be used as a feature. The difference between GPR landmine signals, however, can be observed by comparing the distribution away from the zero intensity, $Z=0$, which is reflected in the second eigenvalue. Namely, as shown in Figure 5, the eigenvalue in the direction of $\mathbf{e}_{2}$ would reflect such a unique pattern, which is used as a feature for a landmine.

3.2. Fourier Transform. Fourier Transform is a way to represent a signal in the time domain as a combination of 




(a)



(b)

FIgURE 4: The GPR data after reducing the ground effects and noise.

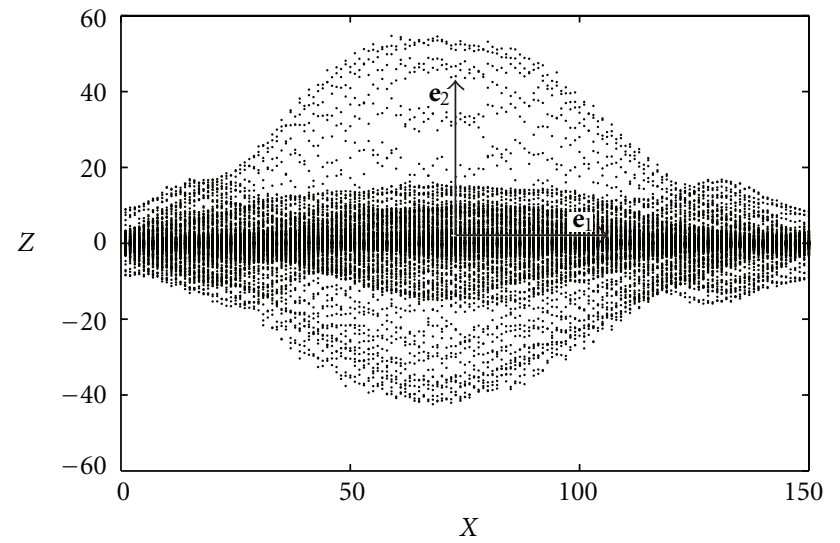

FIGURE 5: GPR data projected onto the $X Z$ plane and eigenvectors of the data set using the PCA method.

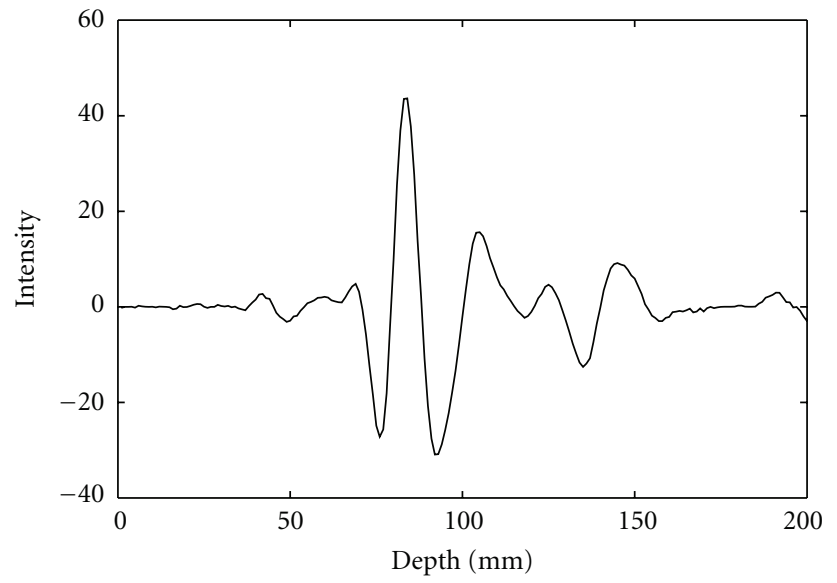

Figure 6: A column of the signal of maximum intensity. frequency components. Consider a column of the GPR data as shown in Figure 6. The signal represented in the frequency domain is in Figure 7 . The components $C_{1}$ and $C_{2}$ at two frequency values, $f_{1}$ and $f_{2}$, respectively, are used as the features of the given signal. The choice of these two frequencies can be made as follows. Consider $l_{a}$ landmines and $l_{b}$ burial conditions. Then, $l_{a} \cdot l_{b}$ GPR signals can be obtained, each of which is transformed to yield $l_{a} \cdot l_{b}$ signals in the frequency domain. Then, the frequencies of the coefficients which can differentiate each landmine as much as possible are chosen after all the signals in the frequency domain are investigated. This approach can be easily extended to include more than two frequency components as features of each landmine.

3.3. Database Construction. A series of experiments for various landmines buried in diverse ground conditions and depths is performed and a database is constructed for storing the features, associated landmine information, and ground conditions.

\section{Landmine Detection and Identification}

Landmine detection is a process to determine whether a signal from GPR contains a landmine, whereas identification includes not only detecting a landmine in the signal but also determining the type of landmine. Therefore, identification is more involved than detection, providing more information about a landmine.

Given a feature extracted from the GPR data, detection and identification is performed by searching for the same feature in the database and retrieving the corresponding landmine information. The possibility that exactly the same feature is found in the database is slim, however, due to various noise and uncertainty contained in the acquired signal. Instead, it is highly likely that a feature close to the given one is found, or multiple landmines with features close to it are returned. Therefore, for robust detection and identification, a systematic approach should be used to eliminate confusion in the decision. In this work, two methods, the counting and Mahalanobis distance-based likelihood ratio test methods, are proposed. 




FIgUre 7: The FT result of the signal.

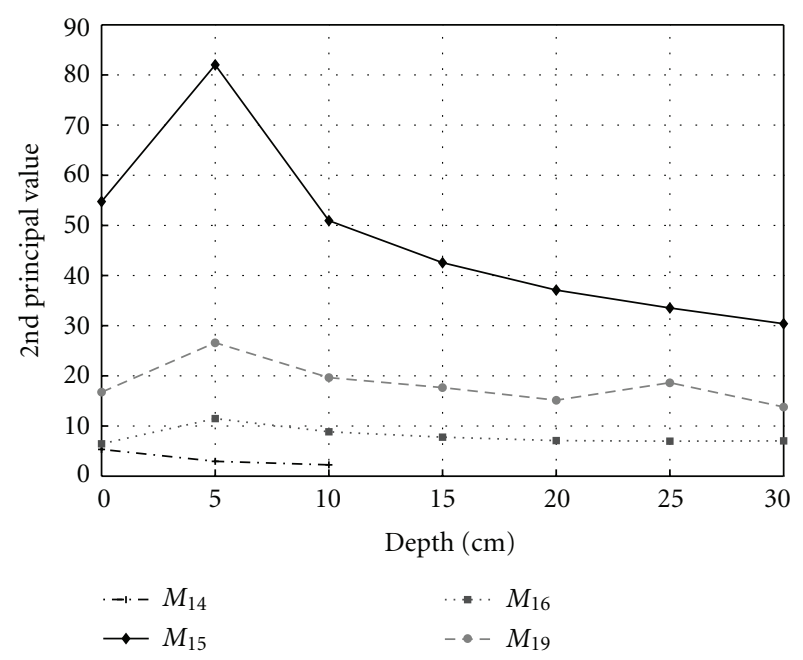

Figure 8: The curves of the second eigenvalues of each landmine with respect to the depth for the dry sand condition.

4.1. Counting Method. The counting method counts the number of matches and chooses the one with the largest number of counts as the identified landmine. Each landmine is given a count variable. A given feature is compared with those in the database. If a match is found, the count variable corresponding to the landmine is increased by one. Here, the matching criterion is to check if the absolute difference between the feature and that in the database is within a given tolerance. There may be multiple matches for one feature. In this case, all of the count variables that match are increased by one. This counting process is performed for all of the features considered. The one with the largest counting value is reported as the detected landmine with its identification information.

4.2. Mahalanobis Distance-Based Likelihood Ratio Test Method. Consider an $n$-dimensional vector $\mathbf{x}=\left(x_{1}, x_{2}, \ldots\right.$, $\left.x_{n}\right)$. The Gaussian probability distribution function for $\mathbf{x}$ is given by

$$
g_{(\mu, \Sigma)}(\mathbf{x})=\frac{1}{\sqrt[n]{2 \pi} \sqrt{|\boldsymbol{\Sigma}|}} e^{-(1 / 2)(\mathbf{x}-\boldsymbol{\mu})^{T} \boldsymbol{\Sigma}^{-1}(\mathbf{x}-\boldsymbol{\mu})} .
$$

Here, $\boldsymbol{\mu}$ is the $n$-dimensional mean vector $\left(E\left[x_{1}\right], E\left[x_{2}\right], \ldots\right.$, $\left.E\left[x_{n}\right]\right)$ and $\boldsymbol{\Sigma}$ the $n \times n$ covariance matrix $\Sigma=\left(\operatorname{Cov}\left[x_{i}, x_{j}\right]\right)$ and $(i, j=1,2, \ldots, n)$. Assume that there are two data groups $G_{1}$ and $G_{2}$ with Gaussian distributions $g_{1}(\mathbf{x})$ and $g_{2}(\mathbf{x})$, respectively. Suppose that $P(\mathbf{x})$ is the probability that an input $\mathbf{x}$ is a landmine and $P\left(\boldsymbol{\omega}_{l}\right)$ is the probability that $\boldsymbol{\omega}_{l}$ is a member of $G_{l}$. If the probability of $\mathbf{x}$ belonging to $G_{1}$, denoted as $P\left(\boldsymbol{\omega}_{1} \mid \mathbf{x}\right)$, is greater than that of $\mathbf{x}$ belonging to $G_{2}, P\left(\boldsymbol{\omega}_{2} \mid \mathbf{x}\right), \mathbf{x}$ is decided to be a member of $G_{1}$. Applying Bayes' theorem, we have

$$
P\left(\boldsymbol{\omega}_{1} \mid \mathbf{x}\right)>P\left(\boldsymbol{\omega}_{2} \mid \mathbf{x}\right) \Longrightarrow \frac{P\left(\mathbf{x} \mid \boldsymbol{\omega}_{1}\right) P\left(\boldsymbol{\omega}_{1}\right)}{P(\mathbf{x})}>\frac{P\left(\mathbf{x} \mid \boldsymbol{\omega}_{2}\right) P\left(\boldsymbol{\omega}_{2}\right)}{P(\mathbf{x})},
$$

which is reduced to

$$
\Lambda(\mathbf{x})=\frac{P\left(\mathbf{x} \mid \boldsymbol{\omega}_{1}\right)}{P\left(\mathbf{x} \mid \boldsymbol{\omega}_{2}\right)}>\frac{P\left(\boldsymbol{\omega}_{2}\right)}{P\left(\boldsymbol{\omega}_{1}\right)} .
$$

The ratio $\Lambda(\mathbf{x})$ is called the likelihood ratio, and the test using this ratio is the likelihood ratio test (LRT). Therefore, an input vector $\mathbf{x}$ is decided to belong to $G_{1}$ if the ratio $\Lambda(\mathbf{x})$ satisfies the condition (4). Otherwise, it belongs to $G_{2}$. In the LRT, the Mahalanobis distances from $\mathbf{x}$ to $G_{k}(k=1,2, \ldots, r)$ are used as input, which is a useful scheme for evaluating similarity between data sets [16]. The distance $D_{M}(\mathbf{x})$ of a vector $\mathbf{x}$ from a data set with mean $\boldsymbol{\mu}$ in $n$-dimension is given by $[16]$

$$
D_{M}(\mathbf{x})=\sqrt{(\mathbf{x}-\boldsymbol{\mu})^{T} \mathbf{S}^{-1}(\mathbf{x}-\boldsymbol{\mu})} .
$$

Here, $\mathbf{S}$ is the pooled covariance matrix of the two data sets, which is the weighted average of the covariance matrices involved.

\section{Experiments}

In this section, a series of experiments is performed to demonstrate the performance of the proposed method. Four types of landmines (two for antihuman, $M_{14}$ and $M_{16}$, and two for antitank, $M_{15}$ and $M_{19}$ ) are used for the experiments. The relative sizes of the landmines are $M_{14}<M_{16}<M_{15}<$ $M_{19} . M_{15}$ and $M_{16}$ are made of metal, while $M_{14}$ and $M_{19}$ are made of plastic. Various ground conditions are prepared in the experiments that consider three moisture levels (dry, moderate, and damp), three materials (sand, soil, and gravel) and seven burial depths (from $0 \mathrm{~cm}$ to $30 \mathrm{~cm}$ with a $5 \mathrm{~cm}$ interval). Each GPR data set is obtained by repeating the scanning 20 times to ensure the accuracy of the scanned data.

5.1. Preparation of GPR Data. The GPR data sets are processed to reduce ground effects from the landmine signals, which are then normalized based on the maximum and minimum signal values. 


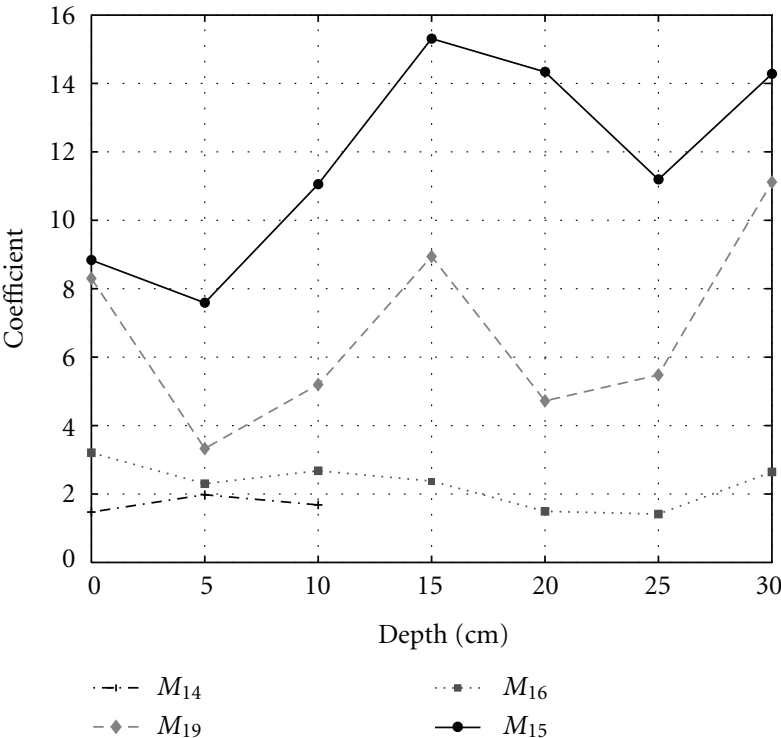

(a)

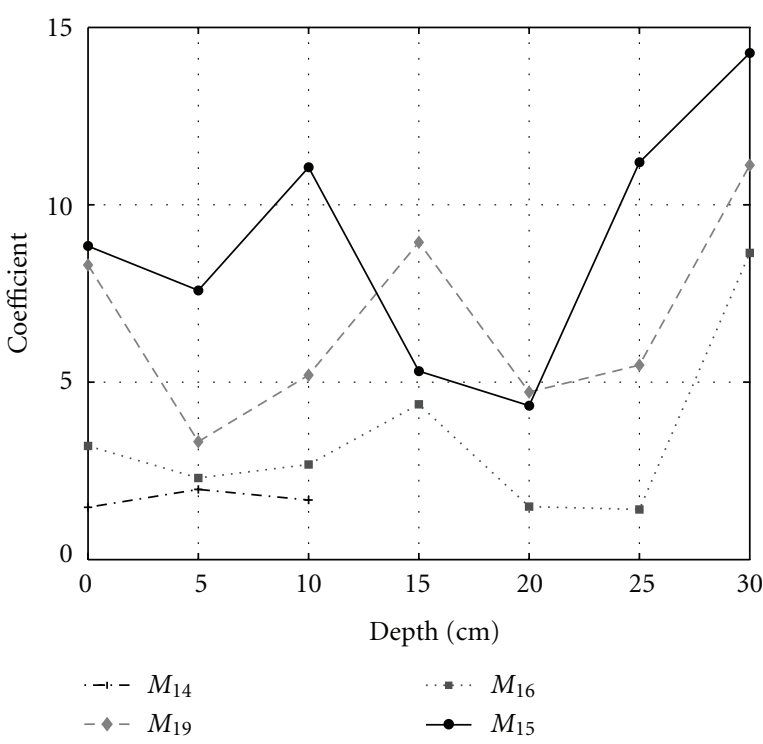

(b)

FIGURE 9: The curves of the Fourier coefficients for (a) $0.71 \mathrm{GHz}$ and (b) $0.95 \mathrm{GHz}$ of the data for each landmine with respect to the depth for the dry sand condition.

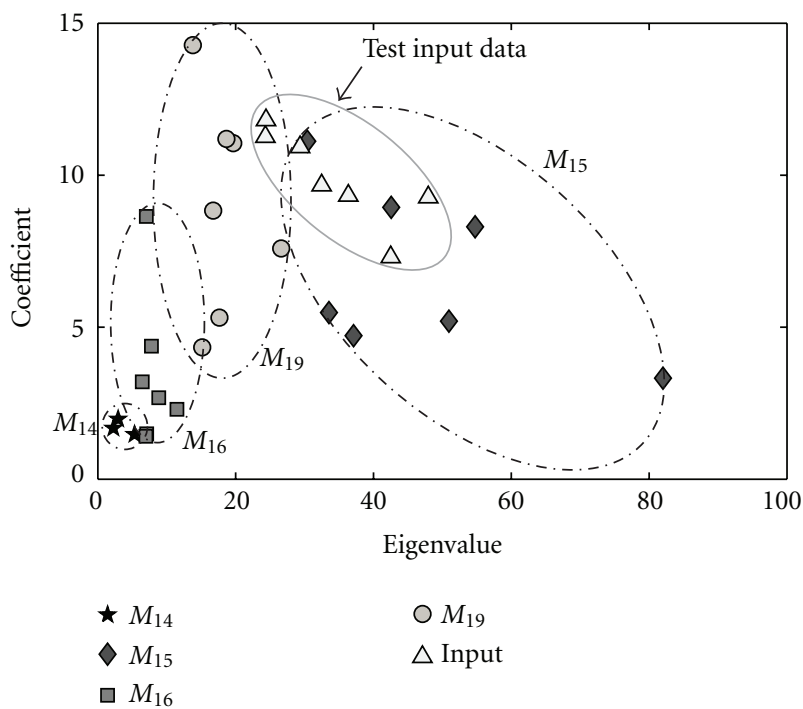

Figure 10: The plots of the second eigenvalues and the Fourier coefficients for $0.71 \mathrm{GHz}$ of each landmine of variable burial depths for the dry sand condition.

5.2. Features. In the experiments, the second eigenvalues from the principal component analysis and the coefficients at two frequencies, $0.71 \mathrm{GHz}$ and $0.95 \mathrm{GHz}$, are selected as features for each landmine. These three features are chosen after checking the differentiability of the landmines considered in the experiment.

For each landmine in all the ground conditions, those three features are computed and stored in the database. Figure 8 shows the variation of the second eigenvalues of each landmine with respect to the depths. Figure 9 shows the variation of the Fourier coefficients at 0.71 and $0.95 \mathrm{GHz}$ with respect to the burial depths for the dry sand condition. Note that the two curves for the $M_{15}$ and $M_{19}$ landmines in Figure 9(b) cross. However, if the eigenvalues and the coefficients at $0.71 \mathrm{GHz}$ are used first, those two landmines can be discriminated. The coefficients at $0.95 \mathrm{GHz}$ are able to differentiate the upper two curves from the rest. The matching tolerance for the counting method is $\pm 5 \%$.

To use the LRT, the second eigenvalues and the coefficients at $0.71 \mathrm{GHz}$ are plotted in a 2D plane as shown in Figure 10, which shows the plot of the second eigenvalues and the coefficients at $0.71 \mathrm{GHz}$ of the four landmines buried at different depths in the dry sand. As illustrated in the figure, each landmine is clustered irrespective of burial depths and the LRT can be successfully applied for landmine identification. If a set of test input data is given as in Figure 10, they can be identified as $M_{15}$ or $M_{19}$ depending on the positions of the input data in the plot.

With the arbitrary noise added to the landmine signals, the proposed method generates satisfactory detection and identification results. Among all of the experiments, one case with landmines buried in sand is presented. Depending on the noise levels added to the signal, from $\pm 1.5 \%$ to $\pm 9.6 \%$, the detection rates are $100 \%$ for all cases, and the identification ratio changes from $100 \%$ to $79.2 \%$, as shown in Figure 11, which illustrates the variation of the identification rates with respect to the noise levels. Additionally, the higher the moisture levels are, the lower the identification rates become. The detection rates for the soil and gravel conditions are also $100 \%$ in the experiments. However, the identification rates vary from approximately $30 \%$ to $100 \%$ depending on the ground condition and the added noise levels. If both 


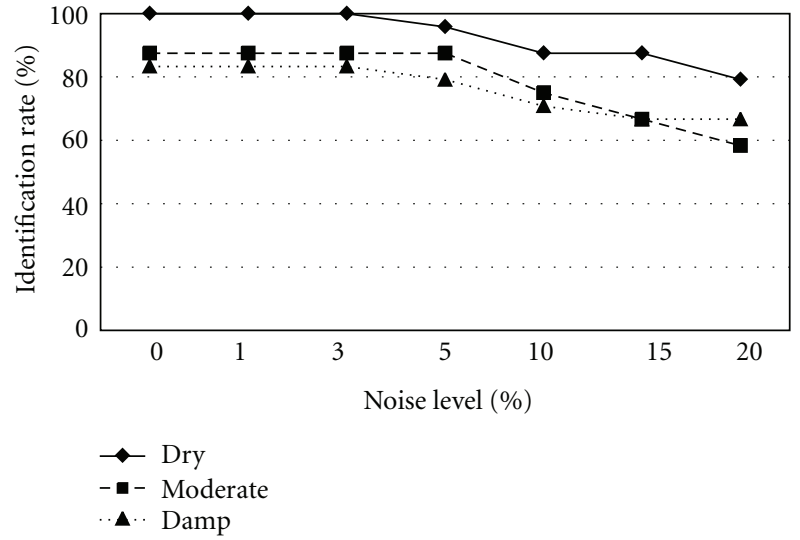

FIgURE 11: The identification rate variation in sand.

methods are compared for each case, the LRT method works better than the counting method for the soil and gravel conditions, whereas the counting method performs better for the cases with signals contaminated by a large amount of noise and landmines buried in sand.

\section{Conclusions}

In this paper, we propose a procedure using data from a GPR to identify landmines buried in the ground of diverse conditions. The proposed method uses multiple features that reflect different aspects of landmines, which enables more stable detection and identification of a landmine. Moreover, using a series of experiments with respect to burial depths, the depth at which an identified landmine exists could also be estimated. Such additional information about a landmine could enhance the landmine removal process.

The proposed method is limited in that it has been tested with only a single landmine case. Therefore, if there are multiple landmines and other foreign objects, the proposed method needs to be refined to handle such cases. The proposed method requires thorough evaluation with field data before it is used in practice. In addition, extending the work to the cases of multiple landmines with diverse objects is recommended for future research.

\section{Acknowledgments}

This work was supported by Unmanned Technology Research Center, Defense Acquisition Program Administration, and Agency for Defense Development.

\section{References}

[1] C. A. Amazeen and M. C. Locke, "Developmental status of the U.S. Army's new handheld standoff mine detection system (HSTAMIDS)," in Proceedings of the 2nd International Conference on the Detection of Abandoned Land Mines, no. 458, pp. 193-197, October 1998.

[2] C. A. Amazeen and M. C. Locke, "US Army's new handheld standoff mine detection system (HSTAMIDS)," in Proceedings of the EUREL International Conference on The Detection of Abandoned Land Mines: A Humanitarian Imperative Seeking a Technical Solution, no. 431, pp. 172-176, October 1996.

[3] J. MacDonald, J. R. Lockwood, J. McFee et al., "Alternatives for landmine detection," Rand Corporation, ch. 2, 2003.

[4] M. Sato, T. Kobayashi, K. Takahashi, J. Fujiwara, and X. Feng, "Vehicle mounted SAR-GPR and its evaluation," in Detection and Remediation Technologies for Mines and Minelike Targets XI, vol. 6217 of Proceedings of SPIE, April 2006.

[5] R. S. Harmon, J. H. Holloway, and J. T. Broach, "Processing of GPR data from NIITEK landmine detection system," in The International Society for Optical Engineering: Detection and Remediation Technologies for Mines and Minelike Targets VIII, vol. 5089 of Proceedings of SPIE, pp. 1375-1382, April 2003.

[6] A. Yarovoy, T. Savelyev, X. Zhuge et al., "Performance of UWB array-based radar sensor in a multi-sensor vehicle-based suit for landmine detection," in Proceedings of the 5th European Radar Conference (EuRAD '08), pp. 288-291, October 2008.

[7] H. Brunzell, "Detection of shallowly buried objects using impulse radar," IEEE Transactions on Geoscience and Remote Sensing, vol. 37, no. 2, pp. 875-886, 1999.

[8] P. D. Gader, M. Mystkowski, and Y. Zhao, "Landmine detection with ground penetrating radar using hidden Markov models," IEEE Transactions on Geoscience and Remote Sensing, vol. 39, no. 6, pp. 1231-1244, 2001.

[9] S. H. Yu, R. K. Mehra, and T. R. Witten, "Automatic mine detection based on ground penetrating radar," in Detection and Remediation Technologies for Mines and Minelike Targets IV, Proceedings of SPIE, pp. 961-972, Orlando, Fla, USA, April 1999.

[10] D. Carevic, "Clutter reduction and target detection in Ground Penetrating Radar data using wavelets," in Detection and Remediation Technologies for Mines and Minelike Targets IV, Proceedings of SPIE, pp. 973-978, Orlando, Fla, USA, April 1999.

[11] C. R. Ratto, P. A. Torrione, and L. M. Collins, "Contextdependent feature selection for landmine detection with ground-penetrating radar," in Detection and Sensing of Mines, Explosive Objects, and Obscured Targets XIV, vol. 7303 of Proceedings of SPIE, pp. 1-12, April 2009.

[12] H. T. Haskett and J. T. Broach, "Automatic mine detection algorithm using ground penetration radar signatures," in Detection and Remediation Technologies for Mines and Minelike Targets IV, Proceedings of SPIE, pp. 942-952, Orlando, Fla, USA, April 1999.

[13] S. L. Tantum, Y. Wei, V. S. Munshi, and L. M. Collins, "A comparison of algorithms for landmine detection and discrimination using ground penetrating radar," in Detection and Remediation Technologies for Mines and Minelike Targets $I V$, vol. 4742 of Proceedings of SPIE, pp. 728-735, Orlando, Fla, USA, April 2002.

[14] V. Kovalenko, Advanced GPR data processing algorithms for detection of anti-personnel landmines [Ph.D. dissertation], Delft University of Technology, 2006.

[15] T. G. Savelyev, L. Van Kempen, H. Sahli, J. Sachs, and M. Sato, "Investigation of time-frequency features for GPR landmine discrimination," IEEE Transactions on Geoscience and Remote Sensing, vol. 45, no. 1, pp. 118-129, 2007.

[16] R. De Maesschalck, D. Jouan-Rimbaud, and D. L. Massart, "The Mahalanobis distance," Chemometrics and Intelligent Laboratory Systems, vol. 50, no. 1, pp. 1-18, 2000. 



Submit your manuscripts at

http://www.hindawi.com
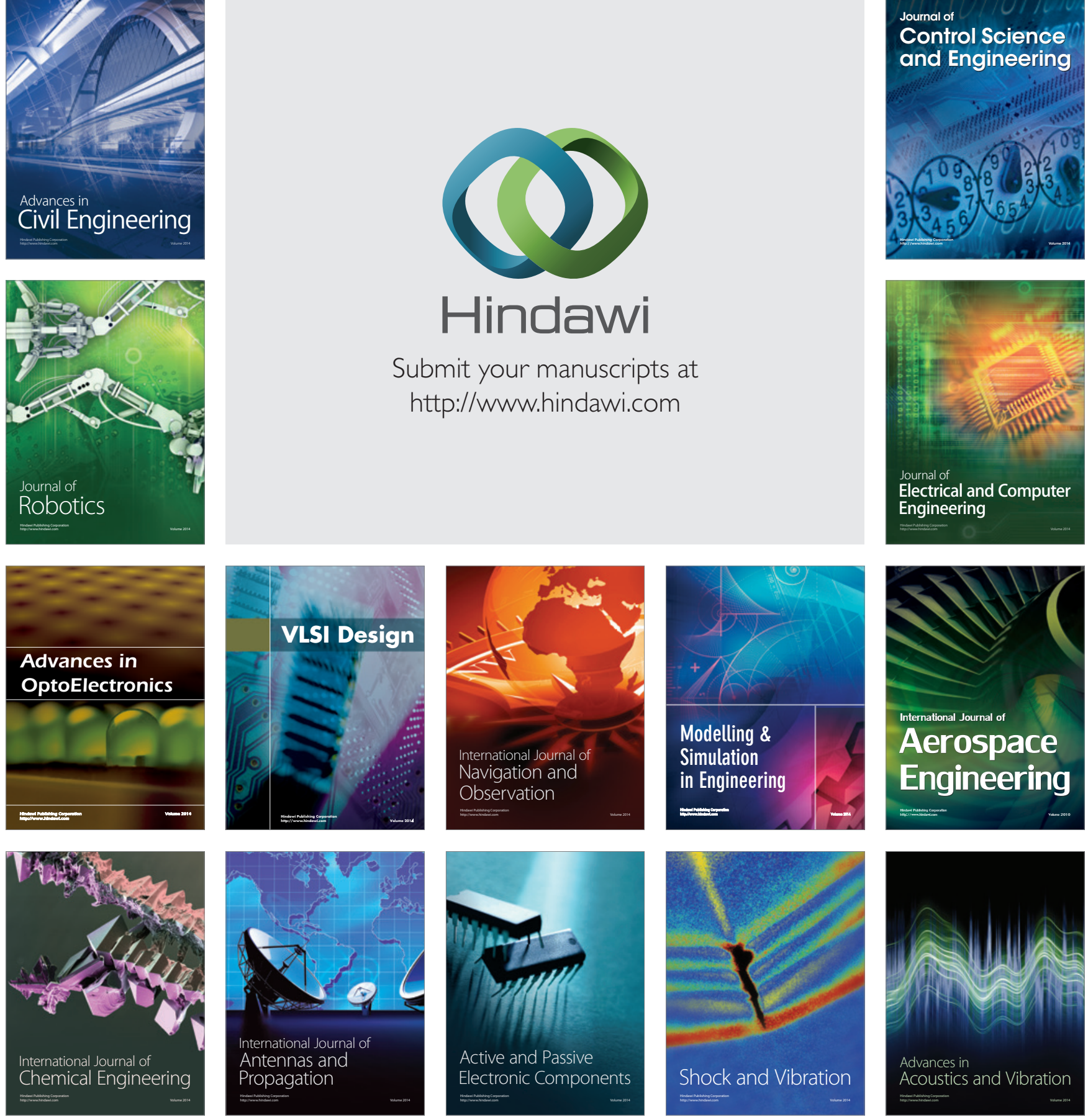